\title{
Memetic Optimized Expectation Maximization Clustering for Energy Effective Data Congregating in WSN
}

\author{
Sridhar Ramaiah ${ }^{1 *}$ \\ Guruprasad Nagraj $^{2}$ \\ ${ }^{1}$ Department of Information Science and Engineering, Global Academy of Technology, Bengaluru, India \\ ${ }^{2}$ Department of Computer Science and Engineering, Global Academy of Technology, Bengaluru, India \\ * Corresponding author's Email: srimln77@gmail.com
}

\begin{abstract}
Clustering is a problem to be resolved for effectively aggregate and transfer information in WSN. Cluster head performs a significant part in the cluster for data accumulation. The energy in wireless network is quite limited. In order to achieve energy efficient data gathering with minimum delay in WSN, a new model called Memetic Optimized Expectation Maximization Clustering (MOEMC) is proposed. The MOEMC model at first computes residual energy for all sensor nodes in network. After that, MOEMC Model designs Dice Similarity Based Expectation Maximization (DS-EM) Clustering algorithm in order to efficiently construct different number of clusters by means of residual energy level. MOEMC Model then employs Annealed Flip Memetic Optimization (AFMO) algorithm to attain enhanced cluster head selection in WSN. The Memetic Optimization algorithm selects an optimal cluster head according to fitness function evaluation for gathering data from its member. Finally, gathered data are routed to sink node via optimal cluster heads in WSN. Hence, MOEMC Model exploit the network lifespan as well as energy proficiency. The MOEMC Model conducts simulation work using various metrics with reverence to a number of data packets and sensor nodes such as Data delivery rate, Packet loss rate, Delay and Energy Usage. From the results, the proposed MOEMC Model achieves energy efficient data gathering process with minimum Packet loss rate and Energy Usage in the wireless sensor networks.
\end{abstract}

Keywords: Cluster head, Expectation maximization, Fitness function, Local search, Memetic, Sensor node, WSN.

\section{Introduction}

A wireless sensor network comprises of lowprice and less-power sensor nodes. All sensor nodes are sited in a precise region and build a wireless network by mode of self-establishing. They assert the adeptness to operate usually at any of the distinct or terrific milieu unlike persons. The data diffusion between nodes is ineffective owed to diverse complex features. Clustering process is used to perform reliable data transmission. The clustering pattern segregates the nodes into several clusters. All clusters in network have distinctive cluster head that sends the data to other sensor nodes in the cluster. A few investigations have been conducted for data congregation process in WSN with diverse techniques. However, energy efficiency during the data gathering was lower. Consequently, MOEMC classic is devised in this research work.

Fitness based Glowworm swarm with Fruitfly Algorithm (FGF) was employed in [1] for increasing network lifespan and energy proficiency in WSN. But, the FGF algorithm does not consider the efficiency of data transmission. Therefore, performance of data delivery rate was not enhanced.Residual Energy-Based Cluster-Head Selection was carried out in [2] using a novel algorithm called R-LEACH [2] for enhancing the throughput and lifetime in WSN. However, energy consumption was more.

An energy balanced QoS based cluster head selection approach was introduced [3] for decreasing computation cost in WSN. But, data loss rate using this strategy was higher. A fuzzy based balanced cost $\mathrm{CH}$ selection algorithm (FBECS) was employed in [4] for improving the performance of data 
gathering in WSN with minimal energy consumption. However, delay using this algorithm was very high.

Cluster-Tree routing scheme for gathering data (CTRS-DG) was designed [5] for cluster creation and $\mathrm{CH}$ selection in WSN. But, optimal cluster head selection was not attained. Energy-aware adaptive kernel density estimation algorithm (EAKDE) was introduced in [6] by means of equalizing the vivacity distribution among the cluster heads in WSN. Nonetheless, energy efficient data aggregation was not obtained.

Attribute-Aware Data Aggregation was achieved in [7] for accomplishing potential-based dynamic routing in WSN with higher accuracy. Nevertheless, energy disbursement amid data collection was very high. Regional Energy Aware Clustering by Isolated Nodes (REAC-IN) was exhibited [8] to perform vitality efficient clustering process. However, packet delivery rate was not at required level.

An Enriched Three-Layer Low-Energy Adaptive Clustering Hierarchy was employed in [9] to accumulate data and route the information. But, the cluster head selection performance was lower. Fuzzy clustering pre-processing and particle swarm optimization was employed in [10] for $\mathrm{CH}$ selection in WSN. However, transmission delay was higher.

To address the above mentioned conventional issues, MOEMC model is developed in this paper. The main contributions of MOEMC model is explained below,

- To enhance the energy efficacy of data congregation in WSN through node clustering when related to state-of-the-art mechanisms, MOEMC model is proposed with the aid of Dice Similarity Based Expectation Maximization (DS-EM) Clustering algorithm and Annealed Flip Memetic Optimization (AFMO) algorithm.

- To attain better node clustering performance in WSN when compared to conventional works, DS-EM Clustering algorithm is devised in MOEMC model. On the divergent to conventional works, EM Clustering process is suggested by using dice similarity calculation to enhance accuracy of node clustering.

- To increase the performance of $\mathrm{CH}$ selection in WSN, Annealed Flip Memetic Optimization (AFMO) is employed in MOEMC model. AFMO is proposed with the aid of annealed selection, distinct crossover and flip mutation.
The residual structure of article is planned as follows: section 2 depicts the related work. Section 3 shows exhaustive process of recommended MOEMC pattern with the aid of architecture diagram. Section 4 illuminates simulation setting. section 5 outlines attained results of suggested MOEMC classic in comparison with two longestablished methods. Decisively, the outcome of the research article is portrayed in section 6 .

\section{Related works}

Multi-criteria based cluster head selection pattern was designed in [11]. But, the performance of delay was not considered.A Pareto optimizationbased approach was developed in [12] for accomplishing clustering and an energy effective, reliable and scalable routing in WSN.In this approach, when a node transmits packets at a high power level, it may generate too much interference in the network and consumes more energy than necessary.Therefore, the minimization of energy utilization is not sufficient.

Energy-aware $\mathrm{CH}$ selection was accomplished in [13] with the application of particle swarm optimization and exploration of packet retransmissions in sensor networks. However, the performance of optimal cluster selection was not effective. A Hybrid Data Collection Approach was presented in [14] to increase the efficiency in WSN with minimal energy and the data collection latency.But, the data delivery rate was not increased.

An enhanced clustering hierarchy $(\mathrm{ECH})$ approach was employed in [15] to achieve energy efficiency in WSNs. However, the network life time was lower.A revised $\mathrm{CH}$ selection process built on LEACH (LEACH-M) was designed [16] to augment the extent of statistics obtained at base station in WSN. But, the energy consumption was not considered.

An Energy Proficient Cluster based Multilevel Hierarchical Routing was introduced in [17] to perform data assortment in multi-hop wireless sensor network. But, the minimization of energy consumption was not sufficient. A TOPSIS Based Cluster Head Selection was accomplished in [18] by using a multi-criteria decision-making technique in WSN. However, the data delivery rate was not enhanced.

Genetic Algorithm Based Optimization was employed in [19] to get better lifetime through optimal sensor placement in WSN's. But, the performance of optimal cluster head selection was not improved. $\mathrm{CH}$ Selection by Arbitrariness with Data Recovery (CHSRDR) scheme was used in [20] 
for choosing the $\mathrm{CH}$ inside the cluster with data recovery.But, the energy efficient cluster selection was not performed.

In order to overcome the above mentioned existing issues such as higher delay and energy utilization, minimum data delivery rate and lower cluster head selection performance, the proposed Memetic Optimized Expectation Maximization Clustering (MOEMC) representative is revived in the WSN for energy effectual data gathering with improved data delivery rate.

\section{Memetic optimized expectation maximization clustering model}

The Memetic Optimized Expectation Maximization Clustering (MOEMC) model is initiated to increase the recitation of energy adequate data gathering in WSN. The MOEMC model is introduced by using Dice Similarity Based Expectation Maximization (DS-EM) Clustering algorithm and Annealed Flip Memetic Optimization (AFMO) algorithm. The architecture schematic of MOEMC paradigm is presented in Fig. 1.

Fig. 1 demonstrates flow processes of MOEMC Model to enrich the energy efficacy of data accumulation in wireless network. As depicted in the above diagram, MOEMC Model primarily calculates residual energy for each sensor node in WSN. Then, MOEMC Model applies Dice Similarity Based Expectation Maximization (DSEM) Clustering algorithm with aim of grouping sensor nodes into consequent cluster.

Subsequently, MOEMC Model applies Annealed Flip Memetic Optimization (AFMO) algorithm with objective of choosing an optimal cluster head $(\mathrm{CH})$ for collecting data from its member. Finally, gathered data are transmitted to sink node through optimal cluster heads in WSN. From that, MOEMC model elevates the data collection accomplishment with a least amount of energy consumption when estimated to conventional mechanisms. The detailed processes of MOEMC prototype are presented below.

\subsection{Dice similarity based expectation maximization clustering}

In MOEMC Model, Dice Similarity Based Expectation Maximization (DS-EM) Clustering algorithm is carried out for node clustering with minimal time in WSN. The DS-EM Clustering algorithm determines probabilities of cluster memberships that depends on one or more

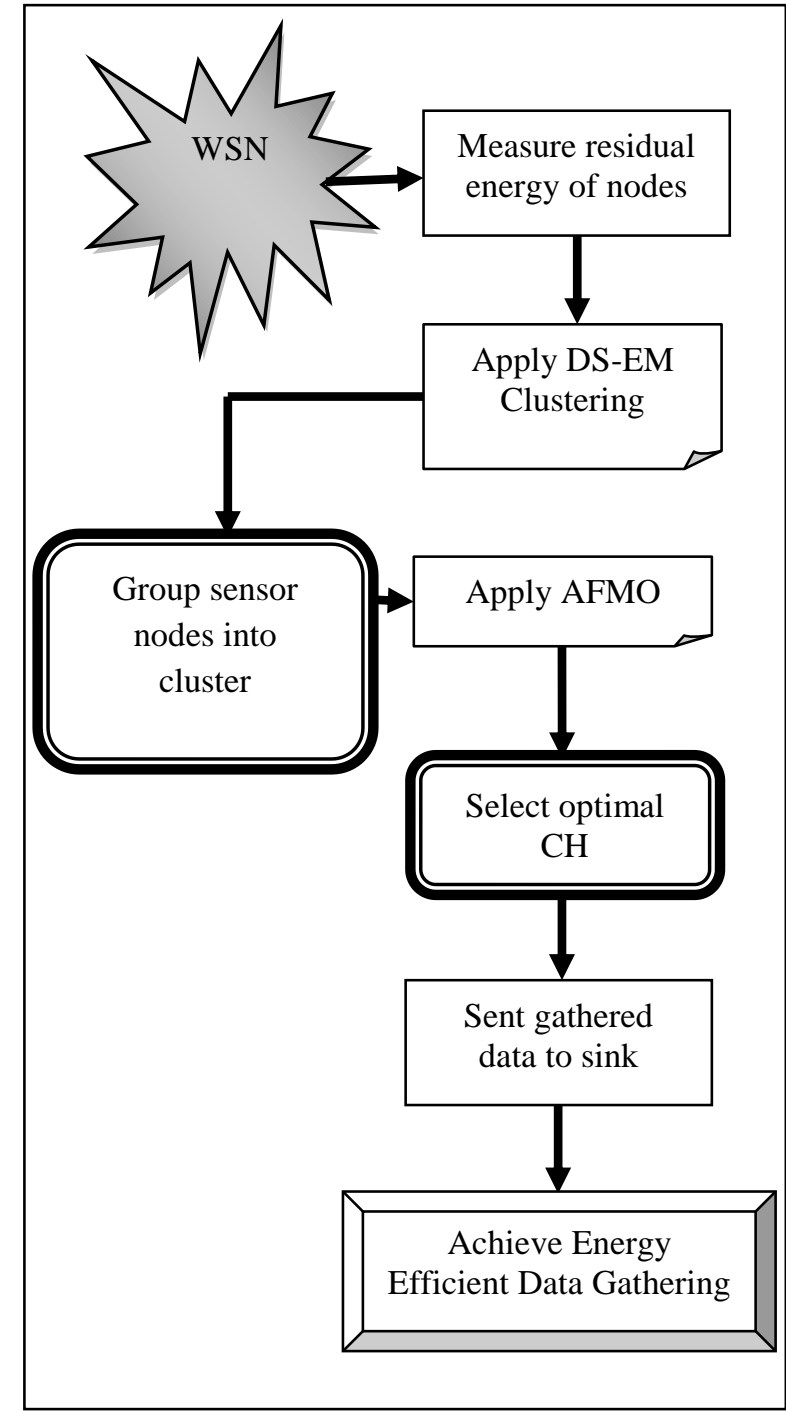

Figure. 1 Architecture diagram of MOEMC model for energy efficient data collection

probability distributions. The goal of the DS-EM Clustering algorithm is to maximize the overall probability or likelihood of the sensor node and thereby forms the final cluster. The block diagram of DS-EM clustering algorithm is shown in Fig. 2.

Fig. 2 shows the overall process of DS-EM Clustering. As depicted in the above figure, DS-EM Clustering initially considers a WSN that comprises many number of sensor nodes denoted as ' $\beta_{1} \beta_{2}, \beta_{3}, \ldots . . \beta_{M}$ '. Here, ' $M$ ' represents the absolute number of nodes. Initially, the number of clusters ' $\alpha_{1}, \alpha_{2}, \alpha_{3}, \ldots . \alpha_{N}$ ' and the cluster centroid ' $\gamma_{1}, \gamma_{2}, \gamma_{3}, \ldots . \gamma_{N}$ ', are initialized in DS-EM clustering algorithm. Followed by, the expected probability between each sensor nodes ' $\beta_{i}$ ' and centroid ' $\gamma_{i}$ ' is computed using below mathematical formula, 


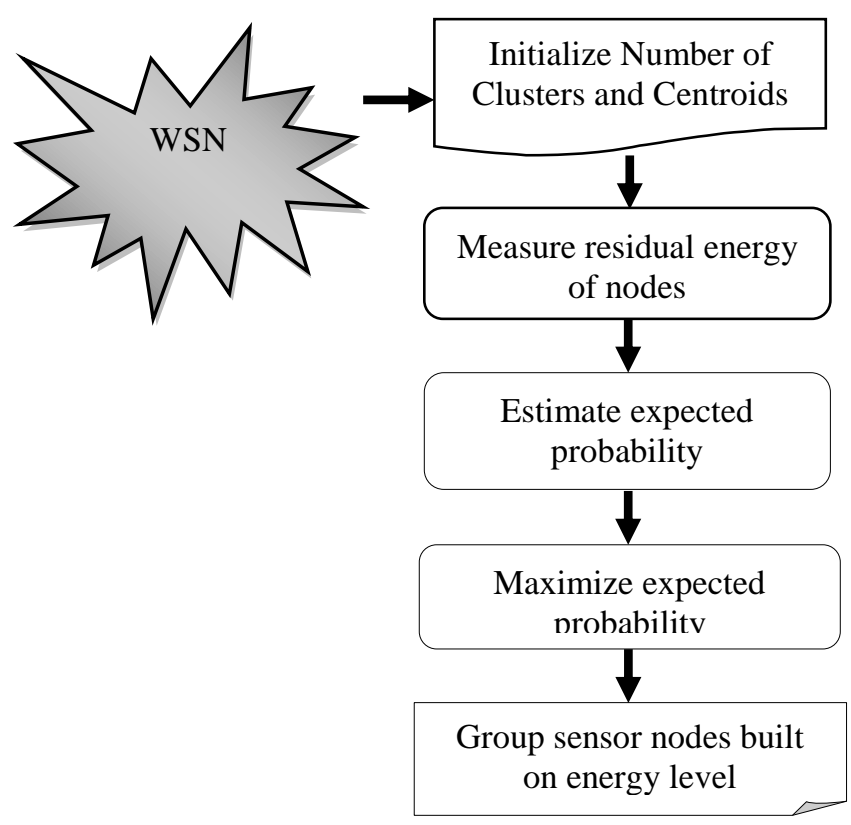

Figure. 2 DS-EM clustering process for efficient cluster formation in WSN

$$
\operatorname{Exp}\left\{P\left(\gamma_{i} \mid \beta_{i}\right)\right\}=\log \left(\prod_{i=1}^{N} \frac{e^{-\frac{1\left(\beta_{i}-k\right)^{2}}{2} z^{2}}}{\sqrt{2 \pi z^{2}}}\right)
$$

From above mathematical Eq. (1), ' $\operatorname{Exp}\left\{P\left(\gamma_{i} \mid \beta_{i}\right)\right\}$ ' designates the expected probability between the sensor nodes ' $\beta_{i}$ ' and centroid ' $\gamma_{i}$ '. Here, ' $k$ ' indicates mean value of cluster centroid and ' $Z$ ' signifies a variance amongst the cluster centroid and sensor nodes. Subsequently DS-EM clustering algorithm calculates the expected probability among each sensor nodes and centroids and thereby clusters all the sensor nodes in wireless network into consequent clusters. Afterward, the DS-EM clustering algorithm predicts the maximization probability to each sensor node to turn out to be a member of the cluster.

In DS-EM clustering, maximization function enhances the expected probability with the assist of MAP computation to group the more related sensor nodes together with a minimal amount of time consumption. Hence, maximum posteriori is mathematically estimated using below,

$$
\begin{aligned}
& \omega_{M A P}=\arg \max \operatorname{Exp}\left\{P\left(\gamma_{i} \mid \beta_{i}\right)\right\} \\
& \omega_{M A P}=\arg \max \log \left(\prod_{i=1}^{M} \frac{e^{-\frac{1\left(\beta_{i}-k\right)^{2}}{2} z^{2}}}{\sqrt{2 \pi z^{2}}}\right)
\end{aligned}
$$

From the above Eqs.(2) and (3), ' $\omega_{M A P}$ ' point outs
Table 1. Tabulation for list of notations

\begin{tabular}{|c|l|}
\hline Notation & \multicolumn{1}{|c|}{ Description } \\
\hline$M$ & Number of nodes \\
\hline$N$ & Number of clusters \\
\hline$\beta_{i}$ & Sensor nodes \\
\hline$\gamma_{i}$ & Centroid \\
\hline$k$ & Mean value \\
\hline$\omega_{M A P}$ & Variance \\
\hline$E x p\left\{P\left(\gamma_{i} \mid \beta_{i}\right)\right\}$ & Maximum a posteriori function \\
\hline$F_{\beta}$ & Memeted probability function \\
\hline$R E_{\beta}$ & Remaining energy level \\
\hline$\varepsilon_{I}$ & Initial energy of sensor nodes \\
\hline$\varepsilon_{C}$ & $\begin{array}{l}\text { Amount of vitality utilized by the } \\
\text { node }\end{array}$ \\
\hline$A S$ & Annealed selection \\
\hline$P\left(F_{i}\right)$ & Probability of the inhabitants \\
\hline$F_{T}$ & Threshold memetic fitness value \\
\hline$n$ & Number of data packets \\
\hline$D D R$ & Data Deliverance Rate \\
\hline$P L R$ & Packet loss rate \\
\hline$D$ & Delay \\
\hline$d_{R T}$ & Data packets acceptance time \\
\hline$d_{S T}$ & Data packet transmitted time \\
\hline ms & milliseconds \\
\hline$E C$ & Energy consumption \\
\hline $\mathrm{J}$ & joule \\
\hline$S S N$ & Single sensor node \\
\hline & \\
\hline
\end{tabular}

the greatest a posteriori function which enhances the projected probability between sensor nodes and centroid to group the associated nodes together grounded on their measured enduring energy. The processes of DS-EM clustering are reiterated until all nodes in the network are congregated into clusters.

The algorithmic process of DS-EM clustering is explained below,

\section{// Dice Similarity Based Expectation // Maximization Clustering Algorithm}

Input: a larger number of sensor nodes ' $\beta_{1}, \beta_{2}, \beta_{3}, \ldots \ldots \beta_{M}$ '

Output: Effective Cluster Formation in WSN

\section{Step 1: Begin}

Step 2: Define the number of Clusters ' $\alpha_{1}, \alpha_{2}, \alpha_{3}, \ldots . \alpha_{N}$ '

Step 3: Define cluster centroids ' $\gamma_{1}, \gamma_{2}, \gamma_{3}, \ldots . \gamma_{N}$ ' Step 4: For each sensor node ' $\beta_{i} \in W S N$ '

Step 5: For each cluster centroid ' $\gamma_{i}$ ' do Step 6: $\quad$ Calculate residual energy level of nodes

Step 7: $\quad$ Evaluate ' $\operatorname{Exp}\left\{P\left(\gamma_{i} \mid \beta_{i}\right)\right\}$ ' using (1) 
Step 8: Find out ' $\omega_{M A P}$ ' using (2) and (3)

Step 9: Group more similar sensor nodes according to residual energy level

\section{Step 10: End for \\ Step 11: End for \\ Step 12:End}

Algorithm 1. Dice similarity based expectation maximization clustering

Algorithm 1, illustrates the step-by-step procedure of DS-EM clustering. Initially, number of ' $M$ ' sensor nodes are randomly deployed in the sensor network. Then, 'N' number of clusters and cluster centroids are initialized. Next, DS-EM clustering significantly forms different number of clusters in sensor network based on their residual energy level by determining maximum a posteriori function. Hence, MOEMC Model increases the performance of node clustering when compared to conventional works.

\subsection{Annealed flip memetic optimization}

In MOEMC Model, Annealed Flip Memetic Optimization (AFMO) algorithm accomplishes data gathering process with lower amount of energy in WSN. The AFMO algorithm is designed by combining genetic algorithm and local search. The AFMO procedure is proposed by using processes such as annealed selection, distinct crossover and flip mutation and fitness value. AFMO algorithm determines optimal sensor nodes as cluster head for achieving energy effective data gathering in WSN. The AFMO process is shown in Fig. 3.

The AFMO algorithm instigates with the initialization of inhabitants by utilizing quantity of sensor nodes in wireless network. AFMO procedure estimates Memetic fitness function for each sensor nodes. Next, the AFMO procedure is repeated for a number of originations awaiting the ending provision is attained. In AFMO algorithm, iteration is pondered as single generation. Throughout each generation, good results are designated from inhabitants with the aid of annealed selection method. Consequently, the distinct crossover operator is used to construct renewed solutions by connecting designated ones. Following crossover, the flip mutation is pertained to the resolutions by arbitrarily altering the solutions. Consequently, a local search procedure is applied in AFMO process to iteratively acquire improved than present solutions for optimal $\mathrm{CH}$ selection in WSN. If the end target is achieved, AFMO procedure will halt

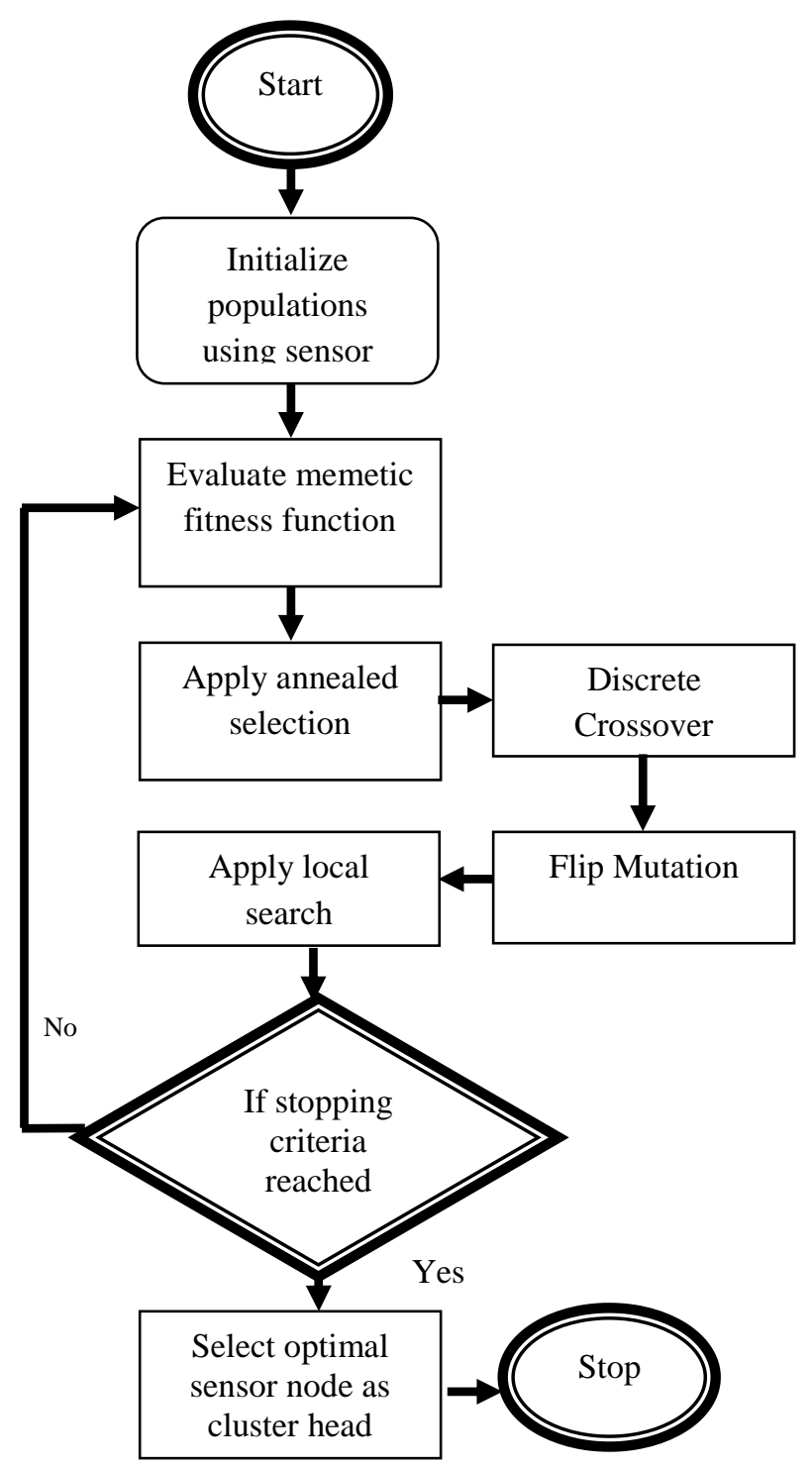

Figure. 3 Flow Processes of AFMO

and the present fair solution is chosen as optimal cluster head.

In AFMO algorithm, primary inhabitants of memes (i.e number of sensor nodes) are set as follows,

$$
\beta_{i}=\beta_{1}, \beta_{2}, \ldots \beta_{n}
$$

From the above mathematical $\mathrm{Eq}$ (4), ' $\beta_{i}$ ' designates a sensor node. Following initialization, Memetic fitness function of each sensor node is determined using below,

$$
F_{\beta}=\left\{R E_{\beta}\right\}
$$

From the above mathematical Eq. (5), ' $F_{\beta}$ ' signifies Memetic fitness function value of sensor node and ' $R E_{\beta}$ ' symbolizes remaining energy level 
of node in network which is mathematically obtained as follows,

$$
R E_{\beta}=\varepsilon_{I}-\varepsilon_{c}
$$

From the mathematical Eq. (6), ' $R E_{\beta}$ ' represents the residual energy of node in wireless network. Here, ' $\varepsilon_{I}$ ' refers the sensor nodes initial energy whereas ' $\varepsilon_{c}$ ' point outs the amount of vitality utilized by the node for gathering data in a network

\section{Annealed Selection:}

In AFMO procedure, Annealed Selection (AS) selects inhabitants from early population. Throughout the Annealed selection course, sensor node with higher Memetic fitness value is selected for subsequent generation. Selection probability for each inhabitant is estimated based on the value of Memetic fitness. Thus, the selection probability of the inhabitants is mathematically obtained using below,

$$
A S=P\left(F_{i}\right)=\frac{F_{\beta}}{\sum_{i=1}^{N} F_{\beta}}
$$

From the above mathematical Eq. (7), ' $F_{i}$ ' denotes mediocre fitness of all inhabitants and ' $N$ ', designates the total number of inhabitants. As a result of Eq. (6), sensor node with greater fitness value is elected in imperative to produce the succeeding generation and accordingly finds best sensor node as $\mathrm{CH}$ within a cluster for attaining energy efficacious data aggregation in WSN.

\section{Discrete Crossover:}

Discrete Crossover (DC) employs arbitrary real number to formulate single infant from two parentages. Discrete Crossover picks two parentages as ' $A$ ' and ' $B$ ' and produce child ' $C$ ' such that it choose memes of both the parentages equally.

\section{Flip Mutation:}

Flip Mutation changes chromosome 0 to 1 and 1 to 0 . In mutation chromosome, for 1 , the subsequent bit in parental gene is flicked ( 0 to 1 and 1 to 0 ) and infant gene is produced.

\section{Local Search:}

Local search is carried out in AFMO algorithm to elect the sensor node with greater fitness value as optimal $\mathrm{CH}$ to collect sensed data from its cluster members. The local search (LS) is mathematically accomplished as,

$$
L S=\left\{\begin{array}{c}
\text { if } F_{\beta}>F_{T}, \quad \beta \text { is optimal } \\
\text { cluster head } \\
\text { otherwise, } \quad \beta \text { is not } \\
\text { optimal cluster head }
\end{array}\right.
$$

From Eq. (8), ' $F_{\beta}$ ' denotes fitness value of sensor node and ' $F_{T}$ ' signifies the threshold memetic fitness value. By the above formula, AFMO process determines sensor node with the largest fitness value as optimum $\mathrm{CH}$.

The algorithmic processes of AFMO is described below,

\section{// Annealed Flip Memetic Optimization // Algorithm}

Input: Clustered sensor nodes

Output: Attain energy effectual data gathering in WSN

\section{Step 1: Start}

Step 2: For every cluster in WSN

Step 3: Initialize the populations using number of sensor nodes

Step 4: Determine' $F_{\beta}$ ' using Eq. (5)

Step 5: Perform annealed selection

Step 6: Accomplish Discrete Crossover

Step 7: Carry out Flip Mutation

Step 8: Apply local search

Step 9: Select optimal sensor node with greater ' $F_{\beta}$ ' as cluster head

Step 10: Cluster head collects data from it members

Step 11: End for

Step 12:Stop

Algorithm 2. Annealed Flip Memetic Optimization

Algorithm 2 presents the step by step progressions of Annealed Flip Memetic Optimization. With the support of the clustered sensor nodes, AFMO Algorithm determines optimal sensor node within cluster with help of fitness function measurement for efficient data cumulation in WSN. If the fitness function of cluster is greater than threshold memetic fitness value, then the cluster is selected as optimal. Otherwise, cluster is not selected as optimal. Therefore, MOEMC Model improves the cluster head selection performance when compared to traditional works.

\section{Simulation settings}

To conclude the performance of suggested MOEMC Model, it is implemented in NS2.34 
Table 2. Simulacrum features

\begin{tabular}{|c|c|}
\hline Simulacrum Features & Assesses \\
\hline Network Simulator & NS 2.34 \\
\hline Square space & 500 \\
\hline $\begin{array}{c}\text { Number of sensor } \\
\text { nodes }\end{array}$ & $\begin{array}{c}\text { Random Waypoint } \\
\text { model }\end{array}$ \\
\hline Mobility standard & $0-20 \mathrm{~m} / \mathrm{s}$ \\
\hline Speed of sensor nodes & $250 \mathrm{sec}$ \\
\hline Simulation time & DSR \\
\hline Protocol & 10 \\
\hline Number of runs & \\
\hline
\end{tabular}

network simulator. MOEMC Model considers 500 sensor nodes distributed in a square area of $A^{2}(1500 \mathrm{~m} \times 1500 \mathrm{~m})$. The different simulation factors are indicated in Table 2.

The efficiency of MOEMC Model is evaluated by means of packet deliverance rate, packet loss rate, delay and consumption of energy. The simulation result of MOEMC Model is compared against traditional FGF [1] and R-LEACH [2].

\section{Results}

In this section, the proportional outcome of MOEMC pattern is exhibited. The performance of MOEMC pattern is assessed against existing FGF [1] and R-LEACH [2] correspondingly. The working of MOEMC Model is determined in conjunction with the below parameters with the aid of tables.

\subsection{Simulation outcomes of data delivery rate}

In MOEMC standard, Data Deliverance Rate 'DDR' is assessed using the below equation,

$$
D D R=\left(\begin{array}{c}
\text { Number of data packets } \\
\text { correctly received }
\end{array}\right) \times 100
$$

From the above Eq. (9), ' $n$ ' implies quantum number of data packets propelled. The data deliverance rate is computed in stipulations of proportion (\%).

\section{Sample computation:}

i. Suggested MOEMC: Number of packets correctly obtained is 22 , and quantity of packets transmitted is 25 . Then the data deliverance rate is attained as follows,

$$
D D R=\frac{22}{25} \times 100=88 \%
$$

ii. Existing FGF: Number of packets exactly obtained is 18 , and quantity of packets transmitted is 25 . Then the data deliverance rate is attained as follows,

$$
D D R=\frac{18}{25} \times 100=72 \%
$$

iii. Existing R-LEACH:Number of packets correctly obtained is 17 , and quantity of packets transmitted is 25 . Then the data deliverance rate is resolved as follows,

$$
D D R=\frac{17}{25} \times 100=68 \%
$$

While considering ' 25 ' packets, the data delivery rate is increased $88 \%$ by using MOEMC, whereas $72 \%$ and $68 \%$ by using existing methods FGF [1] and R-LEACH [2].

Table 3 illustrates the performance outcome of DDR in conjunction with a varied number of packets for three approaches namely MOEMC model, FGF [1] and R-LEACH [2]. The proposed MOEMC model provides better DDR compared to conventional FGF [1] and R-LEACH [2]. This is owing to the application of Dice Similarity Based Expectation Maximization Clustering and Annealed Flip Memetic Optimization on the converse to existing mechanisms. In the proposed MOEMC model, DS-EM clustering process forms the diverse number of clusters based on the residual energy measurement by using maximum a posterior function. Then, the AFMO algorithm is used to find optimal cluster head by use of fitness function calculation. In this way, the proposed MOEMC model efficiently improves data delivery rate by

Table 3. Tabularization outcome for data delivery rate

\begin{tabular}{|c|c|c|c|}
\hline \multirow{2}{*}{$\begin{array}{c}\text { Quantity } \\
\text { of packets }\end{array}$} & \multicolumn{3}{|c|}{ Data deliverance ratio (\%) } \\
\cline { 2 - 4 } & MOEMC & FGF & R-LEACH \\
\hline 25 & 88 & 72 & 68 \\
\hline 50 & 90 & 76 & 74 \\
\hline 75 & 92 & 79 & 80 \\
\hline 100 & 93 & 77 & 79 \\
\hline 125 & 94 & 79 & 81 \\
\hline 150 & 92 & 75 & 77 \\
\hline 175 & 94 & 79 & 82 \\
\hline 200 & 93 & 82 & 84 \\
\hline 225 & 93 & 79 & 81 \\
\hline 250 & 95 & 82 & 84 \\
\hline
\end{tabular}


selecting energy efficient cluster head for packet transmission in WSN.

\subsection{Simulation outcomes of packet loss rate}

In MOEMC prototype, packet loss rate (PLR) of packet is computed using the below expression,

$$
P L R=\left(\begin{array}{c}
\text { Number of data } \\
\text { packets lost }
\end{array}\right) \times 100
$$

From the above $\mathrm{Eq}$ (10), ' $n$ ' implies quantum number of packets remitted. The PLR is estimated in terms of proportion (\%).

\section{Sample computation:}

i. Suggested MOEMC: Number of packets missing is 3 , and number of packets remitted is 25 .The PLR is computed as below,

$$
P L R=\frac{3}{25} \times 100=12 \%
$$

ii. Existing FGF: Number of packets crashed is 7 , and number of packets remitted is 25 . The PLR is determined as below,

$$
P L R=\frac{7}{25} \times 100=28 \%
$$

iii. Existing R-LEACH: Number of packets missing is 8 , and number of packets remitted is 25. The PLR is obtained as below,

$$
P L R=\frac{8}{25} \times 100=32 \%
$$

Let us consider ' 25 ' packets. The proposed MOEMC model reduces the packet loss rate by $12 \%$, where as existing methods FGF [1] and R-LEACH [2] reduces the PLR by $28 \%$ and $32 \%$ respectively.

Table 4 clarifies the performance outcome of PLR in conjunction with a diverged number of packets for three approaches namely MOEMC model, FGF [1] and R-LEACH [2]. The proposed MOEMC model provides better PLR compared to conventional FGF [1] and R-LEACH [2]. This is because of the application of Dice Similarity Based Expectation Maximization Clustering and Annealed Flip Memetic Optimization on the converse to
Table 4. Tabularization outcome for packet loss rate

\begin{tabular}{|c|c|c|c|}
\hline \multirow{2}{*}{$\begin{array}{c}\text { Quantity } \\
\text { of packets }\end{array}$} & \multicolumn{3}{|c|}{ Packet loss ratio (\%) } \\
\cline { 2 - 4 } & MOEMC & FGF & R-LEACH \\
\hline 25 & 12 & 28 & 32 \\
\hline 50 & 10 & 24 & 26 \\
\hline 75 & 8 & 21 & 20 \\
\hline 100 & 7 & 23 & 21 \\
\hline 125 & 6 & 21 & 19 \\
\hline 150 & 8 & 25 & 23 \\
\hline 175 & 6 & 21 & 18 \\
\hline 200 & 7 & 19 & 17 \\
\hline 225 & 7 & 21 & 19 \\
\hline 250 & 5 & 18 & 16 \\
\hline
\end{tabular}

existing mechanisms. From the experimental results analysis, the proposed MOEMC model efficiently reduces the packet loss rate by $66 \%$ when compared to exiting FGF[1] and 64\% when compared to RLEACH [2].

\subsection{Simulation outcomes of delay}

In MOEMC model, delay is computed as the difference amongst the transferring and accepting time of data packets in wireless network. The transmission delay is assessed using the below equation,

$$
D=\left(d_{R T}-d_{S T}\right)
$$

From the above mathematical Eq. (11), ' $D$ ' signifies a delay, ' $d_{R T}$ ' signifies data packets acceptance time, ' $d_{S T}$ ' implies a data packet transmitted time. The delay is influenced in terms of milliseconds (ms).

\section{Sample computation}

i. Suggested MOEMC: Let the number of packets transmitted be 25 , time for accepting the packet is $10 \mathrm{~ms}$ and time for propelling the packet from source node is $0 \mathrm{~ms}$. Consequently the delay is assessed as,

$$
D=10 m s-0 m s=10 m s
$$

ii. Existing FGF: Let the number of packets transmitted be 25 , time for accepting the packet is $14 \mathrm{~ms}$ and time for propelling the packet from source node is $0 \mathrm{~ms}$. Subsequently the delay is deliberated as, 
Table 5. Tabularization outcome for delay

\begin{tabular}{|c|c|c|c|}
\hline \multirow{2}{*}{$\begin{array}{c}\text { Quantity } \\
\text { of packets }\end{array}$} & \multicolumn{3}{|c|}{ Delay (ms) } \\
\cline { 2 - 4 } & MOEMC & FGF & R-LEACH \\
\hline 25 & 10 & 14 & 17 \\
\hline 50 & 12 & 17 & 20 \\
\hline 75 & 14 & 22 & 26 \\
\hline 100 & 21 & 25 & 28 \\
\hline 125 & 23 & 26 & 31 \\
\hline 150 & 25 & 28 & 32 \\
\hline 175 & 27 & 31 & 34 \\
\hline 200 & 31 & 34 & 37 \\
\hline 225 & 34 & 37 & 41 \\
\hline 250 & 35 & 39 & 44 \\
\hline
\end{tabular}

$$
D=14 m s-0 m s=14 m s
$$

iii. Existing R-LEACH: Let the number of packets transmitted be 25, time for accepting the packet is $17 \mathrm{~ms}$ and time for propelling the packet from the source node is $0 m s$. Consequently the delay is assessed as,

$$
D=17 m s-0 m s=17 m s
$$

With the number of ' 25 ' packets, the proposed MOEMC model minimizes the delay by $10 \mathrm{~ms}$, where as $14 \mathrm{~ms}$ and $17 \mathrm{~ms}$ by using existing FGF [1] and R-LEACH [2] methods.

Table 5 clarifies the performance outcome of delay in conjunction to varied number of packets for three approaches namely MOEMC model, FGF [1] and R-LEACH [2]. The proposed MOEMC model provides better delay compared to conventional FGF [1] and R-LEACH [2]. This is due to the application of Dice Similarity Based Expectation Maximization Clustering and Annealed Flip Memetic Optimization on the adverse to existing mechanisms. Therefore, the MOEMC model minimizes the delay by $17 \%$ and $27 \%$ in comparison to existing FGF [1] and RLEACH [2] respectively.

\subsection{Simulation outcomes of energy expenditure}

In MOEMC model, energy consumption ' $E C$ ' determines an extent of vivacity devoured by sensor nodes to gather data in WSN. The utilization of energy is anticipated as follows,

$$
E C=n \times \operatorname{energy}(S S N)
$$

Table 6. Tabularization outcome of energy utilization

\begin{tabular}{|c|c|c|c|}
\hline \multirow{2}{*}{$\begin{array}{c}\text { Total } \\
\text { sensor } \\
\text { nodes }\end{array}$} & \multicolumn{3}{|c|}{ Energy Utilization (J) } \\
\cline { 2 - 4 } & MOEMC & FGF & R-LEACH \\
\hline 50 & 23 & 30 & 35 \\
\hline 100 & 25 & 32 & 38 \\
\hline 150 & 27 & 38 & 42 \\
\hline 200 & 30 & 40 & 46 \\
\hline 250 & 35 & 48 & 55 \\
\hline 300 & 39 & 51 & 60 \\
\hline 350 & 42 & 56 & 67 \\
\hline 400 & 44 & 60 & 72 \\
\hline 450 & 45 & 63 & 77 \\
\hline 500 & 49 & 65 & 80 \\
\hline
\end{tabular}

From the above mathematical Eq. (12), ' energy (SSN)' denotes energy devoured by the single sensor node for collecting data in wireless network, whereas ' $n$ ' represents overall sensor nodes in network. The utilization of energy is calculated by means of joule $(\mathrm{J})$.

\section{Sample computation}

i. Suggested MOEMC: Let the overall sensor nodes be 50 and the vivacity consumed by one sensor nodel is $0.45 J$ oule. The energy consumption is accessed as follows,

$$
E C=50 \times 0.45=23 \mathrm{~J}
$$

ii. Existing FGF: Let the overall sensor nodes be 50 and the vivacity exploited by one sensor node is $0.6 \mathrm{~J}$ oule. The energy consumption is accomplished as follows,

$$
E C=50 \times 0.6=30 \mathrm{~J}
$$

iii. Existing R-LEACH: Let the overall sensor nodes be 50 and the vivacity consumed by one sensor node is 0.7 Joule. The energy consumption is computed as follows,

$$
E C=50 \times 0.7=35 J
$$

Let us assume, the quantum number of sensor nodes be ' 50 '. The energy utilization is reduced $23 \%$ by using MOEMC model, where as $30 \%$ and $35 \%$ by using FGF [1] and R-LEACH [2].

Table 6 illuminates the simulation result of energy utilization in conjunction to a dissimilar quantity of sensor nodes for three mechanisms namely MOEMC scheme, FGF [1] and R-LEACH [2]. The proposed MOEMC model provides better 
energy employment contrasted to conventional FGF [1] and R-LEACH [2]. This is due to the application of Dice Similarity Based Expectation Maximization Clustering and Annealed Flip Memetic Optimization on the adverse to existing mechanisms.From the experimental results evaluation, the proposed MOEMC model reduces the energy utilization during packet transmission in the WSN by $25 \%$ and $36 \%$ when compared to existing FGF[1] and RLEACH [2] respectively.

\section{Conclusion}

In this paper, the MOEMC model is introduced for improving the performance of data assortment in WSN with minimal amount of energy. The objective of MOEMC model is attained by using Dice Similarity Based Expectation Maximization Clustering and Annealed Flip Memetic Optimization algorithms. Initially, the number of cluster and centroids are initialized by using DS-EM Clustering Process. Then, depending on the residual energy the maximum posterior function is calculated for each senor nodes. Depending on the residual energy measurement, all sensor nodes are amassed together into different clusters. Next, with the clustered sensor nodes, AFMO algorithm is performed for selecting optimal cluster head among different cluster heads with help of the fitness function measurement. The performance analysis of MOEMC paradigm is calculated in terms of data deliverance rate, data loss rate, delay, energy consumption and contrasted with two conservative mechanisms such as FGF [1] and R-LEACH [2]. The simulation outcome illustrates that MOEMC scheme delivers improved data aggregation recitation with an improvement of data delivery rate and depreciation of energy utilization when contrasted such as to contemporary mechanisms. However, the proposed MOEMC scheme lacks in terms of secure data transmission. It does not consider any malicious activity during data packet transmission in the WSN. In future, the proposed work is further extended to achieve energy efficient with secure data transmission in wireless network with application of new developed algorithms.

\section{Conflicts of Interest}

The authors declare no conflict of interest.

\section{Author Contributions}

Conceptualization, Sridhar Ramaiah and Guruprasad Nagraj; methodology, Sridhar Ramaiah and Guruprasad Nagraj; Software, Sridhar Ramaiah; formal analysis, Sridhar Ramaiah and Guruprasad Nagraj; investigation, Sridhar Ramaiah and Guruprasad Nagraj; writing-original draft preparation, Sridhar Ramaiah; writing-review and editing, Guruprasad Nagraj; supervision, Guruprasad Nagraj; project administration, Sridhar Ramaiah and Guruprasad Nagraj.

\section{References}

[1] K. N. Dattatraya and K. R. Rao, "Hybrid based cluster head selection for maximizing network lifetime and energy efficiency in WSN", Journal of King Saud University - Computer and Information Sciences, pp. 1-11, 2019.

[2] T. M. Behera, S. K. Mohapatra, U. C. Samal, M. S. Khan, M. Daneshmand, and A. H. Gandomi, "Residual Energy Based Cluster-head Selection in WSNs for IoT Application", IEEE Internet of Things Journal, Vol. 6, No. 3, pp. 5132 5139, 2019.

[3] S. Mahajana, J. Malhotra, and S. Sharm, "An energy balanced QoS based cluster head selection strategy for WSN", Egyptian Informatics Journal, Elsevier, Vol. 15, No. 3, pp. 189-199, 2014.

[4] P. S. Mehra, M. Najmud, and D. B. Alam, "Fuzzy based enhanced cluster head selection (FBECS) for WSN", Journal of King Saud University - Science, Elsevier, Vol. 32, No.1, pp. 390-401, 2020.

[5] W. Osamy, A. M. Khedr, A. Aziz, and A. A. El-Sawy, "Cluster-Tree Routing Based Entropy Scheme for Data Gathering in Wireless Sensor Networks", IEEE Access, Vol. 6, pp. 77372 77387, 2018.

[6] F. Liu and Y. Chang, "An Energy Aware Adaptive Kernel Density Estimation Approach to Unequal Clustering in Wireless Sensor Networks", IEEE Access, Vol. 7, pp. 40569 40580, 2019.

[7] F. Ren, J. Zhang, Y. Wu, T. He, C. Chen, and C. Lin, "Attribute-Aware Data Aggregation Using Potential-Based Dynamic Routing in Wireless Sensor Networks", IEEE Transactions on Parallel and Distributed Systems, Vol. 24, No. 5, pp. $881-892,2013$.

[8] J. S. Leu, T.H. Chiang, M. C. Yu, and K. W. Su, "Energy Efficient Clustering Scheme for Prolonging the Lifetime of Wireless Sensor Network with Isolated Nodes", IEEE Communications Letters, Vol. 19, No. 2, pp. $259-262,2015$.

[9] J. S. Lee and T. Y. Kao, "An Improved ThreeLayer Low-Energy Adaptive Clustering 
Hierarchy for Wireless Sensor Networks", IEEE Internet of Things Journal, Vol. 3 , No. 6 , pp. $951-958,2016$.

[10] Q. Ni, Q. Pan, H. Du, C. Cao, and Y. Zhai, “A Novel Cluster Head Selection Algorithm Based on Fuzzy Clustering and Particle Swarm Optimization", IEEE/ACM Transactions on Computational Biology and Bioinformatics, Vol. 14, No. 1, pp. 76-84, 2017.

[11] H. Farman, B. Jan, H. Javed, N. Ahmad, J. Iqbal, M. Arshad, and S. Ali, "Multi-criteria based zone head selection in Internet of Things based wireless sensor networks", Future Generation Computer Systems, Vol. 87, pp. 364-371, 2018.

[12] R. Elhabyan and W. M. St-Hilaire, "A Pareto optimization-based approach to clustering and routing in Wireless Sensor Networks", Journal of Network and Computer Applications, Elsevier, Vol. 114, pp. 57-69, 2018.

[13] B. Singha and D. K. Lobiyala, "Energy-aware cluster head selection using particle swarm optimization and analysis of packet retransmissions in WSN", Procedia Technology, Vol. 4, pp. 171 - 176, 2012.

[14] R. Zhang, J. Pan, D. Xie, and F. Wang, "NDCMC: A Hybrid Data Collection Approach for Large-Scale WSNs Using Mobile Element and Hierarchical Clustering", IEEE Internet of Things Journal, Vol. 3, No. 4, 2016.

[15] H. E. Alami and A. Najid, "ECH: An Enhanced Clustering Hierarchy Approach to Maximize Lifetime of Wireless Sensor Networks", IEEE Access, Vol. 7, pp. 107142 - 107153, 2019.

[16] L. Zhao, S. Qu, and Y. Yi, "A modified clusterhead selection algorithm in wireless sensor networks based on LEACH", EURASIP Journal on Wireless Communications and Networking, Springer, Vol. 2018, No. 287, pp. 1-8, 2018.

[17] K. Kumaresan and S. NithyaKalyani, "Energy Efficient Cluster based Multilevel Hierarchical Routing for multi-hop Wireless Sensor Network", Journal of Ambient Intelligence and Humanized Computing, Springer, 2020.

[18] F. Hamzeloei, M. K. Dermany, "A TOPSIS Based Cluster Head Selection for Wireless Sensor Network", Procedia Computer Science, Vol. 98, pp. 8-15, 2016.

[19] T. Ganesan and P. Rajarajeswari, "Genetic Algorithm Based Optimization to Improve the Cluster Lifetime by Optimal Sensor Placement in WSN's", International Journal of Innovative Technology and Exploring Engineering (IJITEE), Vol. 8, No. 8, pp. 3400-3408, 2019.
[20] D. P. Singh, R. H. Goudar, B. Pant, and S. Rao, "Cluster head selection by randomness with data recovery in WSN", CSI Transactions on ICT, Vol. 2, pp. 97-107, 2014. 